ACCEPTED TO MNRAS

Preprint typeset using $\mathrm{LAT}_{\mathrm{E}} \mathrm{X}$ style AASTeX6 v. 1.0

\title{
NON-ZEEMAN CIRCULAR POLARIZATION OF MOLECULAR SPECTRAL LINES IN THE ISM
}

\author{
Mohammed Afif Chamma ${ }^{1}$, Martin Houde ${ }^{1}$, Josep Miquel Girart ${ }^{2,3}$ and Ramprasad RaO ${ }^{4}$ \\ ${ }^{1}$ Department of Physics and Astronomy, The University of Western Ontario, London, ON, N6A 3K7, Canada \\ ${ }^{2}$ Institut de Ciències de l'Espai (ICE, CSIC), Can Magrans, S/N, E-08193 Cerdanyola del Vallès, Catalonia, Spain \\ ${ }^{3}$ Institut d'Estudis Espacials de Catalunya (IEEC), E-08034, Barcelona, Catalonia \\ and \\ ${ }^{4}$ Submillimeter Array, Academia Sinica Institute of Astronomy and Astrophysics, 645 N. Aohoku Place, Hilo, HI 96720, USA
}

(Received May 14 2018; Revised July 11 2018; Accepted July 30 2018)

\begin{abstract}
Accurately measuring the magnetic field in the ISM is essential for understanding star-formation processes. We searched archival data of the Submillimeter Array (SMA) for evidence of circular polarization in common molecular tracers, most notably CO. This circular polarization possibly arises from anisotropic resonant scattering, which would imply that some background linearly polarized radiation is being converted to circular polarization. We find circular polarization in the star-forming regions NGC7538 (in $\mathrm{CO}$ ) and Orion $\mathrm{KL}$ (in $\mathrm{CO}$ and $\mathrm{SiO}$ ), as well as in the carbon star IRC+10216 (in $\mathrm{CS}, \mathrm{SiS}, \mathrm{H}^{13} \mathrm{CN}$ and $\mathrm{CO}$ ) at high enough levels to suggest that the presence of circular polarization in these spectral lines is common for such objects. This implies that measuring circular polarization is important when studying magnetic fields through the linear polarization of molecular spectral lines in the interstellar medium. We also provide a simple Python wrapper for the Miriad data reduction package.

Keywords: circumstellar matter - ISM: clouds - polarization - magnetic fields
\end{abstract}

\section{INTRODUCTION}

Understanding the role of magnetic fields in starforming regions allows us to test ideas about freefall collapse and support mechanisms in molecular clouds, filling in details about the star formation process. By measuring the radiation from starforming regions astronomers use polarimetry to infer the magnitude and orientation of the magnetic field. The Davis-Chandrasekhar-Fermi (DCF) method (Davis 1951; Chandrasekhar \& Fermi 1953) uses the dispersion of polarization angles (PA) of linear polarization (LP) for measuring the plane-of-the-sky component of the magnetic field. The presence of a magnetic field leads to LP radiation because dust and molecules will align themselves relative to the field. Aligned particles can emit radiation with a net level of LP greater than zero. Aligned dust can also absorb radiation whose polarization is aligned with its long axis, acting as a sort of polarizing grid. Thus measuring the amount of dust LP in the infrared continuum tells us about the degree to which the dust is aligned with the magnetic field, which in turn can tell us about the strength of the mag- netic field (Davis 1951; Chandrasekhar \& Fermi 1953; Crutcher 2012).

The alignment of molecules and their interaction with the ambient magnetic field can cause their transitions to be linearly polarized by a few percent through the socalled Goldreich-Kylafis (GK) effect (Goldreich \& Kylafis 1981). The GK effect can occur for molecular lines with even weak Zeeman splitting when the radiation field (for example) is anisotropic and the splitting frequency is greater than the collisional rate and other radiative processes. These conditions can cause a population imbalance in the magnetic sublevels that results in a net LP aligned either perpendicular or parallel to the plane-of-the-sky component of the magnetic field. LP due to the GK effect was first detected in CS $(J=2 \rightarrow 1)$ and $\operatorname{HCN}(J=1 \rightarrow 0)$ in IRC+10216 and CRL 2688 by Glenn et al. (1997) and later in CO $(J=3 \rightarrow 2)$ and $(J=2 \rightarrow 1)$ by Greaves et al. (1999). Shortly after, LP from the GK effect was detected interferometrically for the first time and used to map the direction of the magnetic field in NGC1333 IRAS4A (Girart et al. 1999). As with dust, the PA associated with 
the LP in the spectral line can be measured and used to infer properties of the magnetic field through a dispersion analysis (Davis 1951; Chandrasekhar \& Fermi 1953; Crutcher 2012). Observations in DR 21(OH) of LP in $\mathrm{CO}(J=2 \rightarrow 1)$ were compared with simultaneous dust continuum polarization measurements to assess the accuracy in using the GK effect to probe the magnetic field (Lai et al. 2003; Cortes et al. 2005).

For molecular lines circular polarization (CP) is usually ignored, largely because of difficulty in its measurement and its assumed irrelevance. However, a significant amount of unexpected circular polarization was reported by Houde et al. (2013) in $\mathrm{CO}(J=2 \rightarrow 1)$, a common tracer through LP of magnetic fields (Crutcher 2012), using FSPPol (Hezareh et al. 2010) at the Caltech Submillimeter Observatory (CSO). The presence of $\mathrm{CP}$ in a molecular transition can be explained with Zeeman splitting for some molecules/transitions possessing a significant magnetic moment (e.g., CN), but $\mathrm{CO}$ is highly insensitive to the Zeeman effect. In addition, the observed Stokes $V$ profile in Orion KL was positive and symmetric, which is also unexpected since Zeeman splitting often gives rise to an approximately antisymmetric Stokes $V$ profile. To explain this detection a model was proposed whereby background LP radiation is converted to $\mathrm{CP}$ radiation through anisotropic resonant scattering (ARS) (Houde et al. 2013; Houde 2014). This was further tested in the supernova remnant IC443 by Hezareh et al. (2013), where the measured CP flux of CO lines $(J=2 \rightarrow 1)$ and $(J=1 \rightarrow 0)$ were 're-inserted' into the measured LP flux to account for the LP-to-CP conversion and its effect on the PA. They found that the PAs obtained from the different CO transitions only agreed with each other and with those obtained from dust polarimetry after the CP flux was accounted for. If ARS is common to other objects then using LP in CO as a tracer of the magnetic field will introduce a systematic error unless the CP of CO lines is also measured.

The main goal of this paper is to find further evidence of CP in more objects and molecular lines through a search of archival data of the Submillimeter Array (SMA). In Section 2 we discuss the issues that arise when doing polarimetry with radio interferometry, focusing specifically on circular polarization (CP). Section 3 will give in detail our scheme for correcting a spurious source of CP that arises with the SMA. Section 4 presents archival observations of four objects made with the SMA on Mauna Kea and investigates the reliability of our CP detections. Finally, in Section 5 we will highlight the significance of these CP detections and summarize their implications.

\section{MEASUREMENT OF CP WITH RADIO INTERFEROMETRY}

The measurement of CP is challenging to calibrate, especially when using radio interferometers like the SMA or ALMA. The SMA, which has linear feeds, uses a quarter-waveplate (QWP) in the path of the antenna beam to convert incident LP light to CP light to measure LP signals before sending them to the correlator. While not its most common use at the SMA the QWP can work the other way to measure $\mathrm{CP}$ : incident $\mathrm{CP}$ is converted to LP and then measured by the receivers. On the other hand, ALMA which also has linear feeds does not use a QWP and measures LP signals directly. While both measurement techniques can be used to measure CP, the calibration process is different (Sault et al. 1996). Despite the calibration challenges the SMA has been used to take precise measurements of CP from dust continuum in Sgr A* (Muñoz et al. 2012). Additionally, the VLBA, equipped with circular feeds, has been used to take full Stokes $I, Q, U$, and $V$ measurements of $\mathrm{SiO}$ masers at 43.1 and $42.8 \mathrm{GHz}$ (Cotton et al. 2011). In that work a correction for spurious CP analogous to the correction detailed in Section 3 is performed for accurate calibration of Stokes $V$.

For a discussion on measuring $\mathrm{CP}$ with radio interferometry and on design choices at the SMA (such as the choice of converting from LP-to-CP and vice-versa) see Hamaker et al. (1996) and Marrone et al. (2008).

\subsection{Linear vs. Circular Feeds}

To illustrate briefly the differences between the two feed types consider the following: with an orthogonal CP basis, the Stokes $V$ parameter for a beam of radiation is defined by $V \propto\left\langle E_{R}^{2}\right\rangle-\left\langle E_{L}^{2}\right\rangle$, where $E_{R}$ and $E_{L}$ are the right-CP and left-CP electric fields, respectively. With an orthogonal LP basis Stokes $V$ is defined by $V \propto-2 \operatorname{Im}\left(E_{x} E_{y}^{*}\right)$, where $E_{x}$ and $E_{y}$ are the LP fields, and $\operatorname{Im}()$ denotes the imaginary part. In the $\mathrm{CP}$ basis case we take the difference of two measured intensities, while the linear feed case requires us to measure the phase of the electromagnetic wave.

Now, when the measurement is made with interferometry it is the visibilities - the correlated waveforms between a pair of antennae - that are measured. In the CP basis case the Stokes $V$ visibility scales as $\mathcal{V}_{\mathrm{V}} \propto \mathcal{V}_{R R}-\mathcal{V}_{L L}$, where $\mathcal{V}_{R R}$ and $\mathcal{V}_{L L}$ are the visibilities obtained from correlating two antennae measuring right-CP and left-CP, respectively (see eq. (3) below). In the linear feed case the Stokes $V$ visibility is coupled with the Stokes $Q$ and $U$ visibilities (see Section 4.1 of Thompson et al. 2001). This means the Stokes $Q$ and $U$ of any calibration object must be measured as well. This is not possible with the SMA setup used for the observations discussed here.

The SMA uses observations of a bright point source over a large range of parallactic angles to distinguish 
between source and instrumental polarization and determine the polarization leakage terms. Determining these leakage terms is crucial for accurate Stokes $Q$ and $U$ measurements, however Stokes $I$ and $V$ are independent of the leakage terms to first-order (Thompson et al. 2001; Marrone et al. 2008; Muñoz et al. 2012).

\section{SQUINT CORRECTION}

We now describe spurious Stokes $V$ signals that can arise during observations such as those presented here and our scheme for correcting them. This instrumental Stokes $V$ comes from a slight pointing offset between the left- and right-handed CP beams.

The archival data used were in all cases observed with the goal of measuring LP (i.e., the Stokes $Q$ and Stokes $U$ parameters). As mentioned earlier on the SMA this is done with a QWP placed in front of the LP receivers to convert incident $\mathrm{CP}$ (LP) to LP (CP). While this method suffers from the errors that arise when subtracting two large measurements from each other, when performing $\mathrm{CP}$ measurements, it avoids having to solve for the LP terms of calibration objects (Marrone et al. 2008; Thompson et al. 2001).

The output of the interferometer is the visibility, which consists of the cross-correlation between the voltage signals from a pair of antennae and can be written as

$$
\mathcal{V}(u, v)=\frac{\left\langle V_{a}(t) \star V_{b}(t)\right\rangle}{A_{0} \Delta \nu},
$$

where $A_{0}$ is the collecting area of the antennae, $\Delta \nu$ is the bandwidth, and $\left\langle V_{a}(t) \star V_{b}(t)\right\rangle$ is the time-averaged cross-correlation of voltage signals $V_{a}(t)$ and $V_{b}(t)$ from antennae $a$ and $b$, respectively. The arguments $u$ and $v$ are determined by the baseline separation and orientation of the two antennae. Thus a pair of antennae samples a single point of the visibility function $\mathcal{V}(u, v)$ (Thompson et al. 2001).

Through the van Cittert-Zernike theorem it can be shown that the visibility function $\mathcal{V}(u, v)$ gives the Fourier transform of the source intensity $I(l, m)$ for angular position $l$ and $m$ (chap. 3 of Thompson et al. 2001). Thus we have

$$
\mathcal{V}(u, v)=\iint e^{-i u l} e^{-i v m} I(l, m) d l d m .
$$

In practice the true source intensity $I(l, m)$ is convolved with the instrumental response to a point source, called the instrumental beam, and a deconvolution step is necessary to obtain the true map.

Given antennae $a$ and $b$, the Stokes $V$ visibility in the circular feed case is given by (Thompson et al. 2001; Muñoz et al. 2012)

$$
\mathcal{V}_{V} \simeq \frac{1}{2}\left\{\mathcal{V}_{R R} /\left(g_{R a} g_{R b}^{*}\right)-\mathcal{V}_{L L} /\left(g_{L a} g_{L b}^{*}\right)\right\},
$$

where $\mathcal{V}_{R R}$ and $\mathcal{V}_{L L}$ are the right-handed CP and lefthanded $\mathrm{CP}$ visibilities, respectively, measured by appropriately orienting the QWP that is placed in the beam of the antennae and correlating their responses (Marrone et al. 2008). The complex gain factors for each polarization for each antenna are $g_{R a}, g_{R b}, g_{L a}$ and $g_{L b}$ where $R$ and $L$ are for right- and left-CP, respectively. Because the Stokes $V$ visibility is found by taking the difference of two beams a slight offset gives rise to pairs of positive and negative peaks of Stokes $V$, as can be seen in Figure 1 , for example. The cause of this offset is uncertain, but possibly arises due to slight differences in the index of refraction of the QWP when it is rotated.

To correct this offset we first note that since the visibilities are the Fourier transform of the intensity map (eq. 2), an offset in image space results in a complex factor in visibility space that can be absorbed into the gain coefficients. To see this, consider a map $I(l, m)$ that represents the true intensity $I$ at angular position $l$ and $m$. If the instrument introduces an arbitrary offset to position $\left(l_{0}, m_{0}\right)$, then the final image we calculate is shifted such that $I^{\prime}(l, m)=I\left(l-l_{0}, m-m_{0}\right)$ and the measured visibility of the shifted map becomes

$$
\begin{aligned}
\mathcal{V}^{\prime}(u, v) & =\iint e^{-i u l} e^{-i v m} I\left(l-l_{0}, m-m_{0}\right) d l d m \\
& =\iint e^{-i u\left(\alpha+l_{0}\right)} e^{-i v\left(\delta+m_{0}\right)} I(\alpha, \delta) d \alpha d \delta \\
& =e^{-i u l_{0}} e^{-i v m_{0}} \iint e^{-i u \alpha} e^{-i v \delta} I(\alpha, \delta) d \alpha d \delta \\
& =e^{-i u l_{0}} e^{-i v m_{0}} \mathcal{V}(u, v) \\
& \equiv g_{\text {offset }} \mathcal{V}(u, v)
\end{aligned}
$$

where we used the change of variables $\alpha=l-l_{0}$ and $\delta=m-m_{0}$. We thus find the aforementioned complex factor that multiplies the true visibility $\mathcal{V}(u, v)$. It is therefore easiest to correct for the offset in visibilityspace by using Miriad (Sault et al. 1995) to solve for the gain coefficients on each of the two polarized beams, independently of each other. Specifically the process is:

1. The observations are calibrated for gain and phase in the usual way using observations of known sources (usually quasars like 3C84, 3C454, etc.; see Miriad User Guide (Sault et al. 2008)).

2. The visibilities are split into line-free continuum and line data that are then mapped to obtain models using the 'clean' algorithm (Sault et al. 2008).

3. The separate continuum and line data are further split into $L L$ and $R R$ visibilities. Miriad's selfcal is used on the continuum data to solve for the gain coefficients of each antenna and each polarization $(L$ or $R$ ). This is done by minimizing the 
difference between measured visibilities $\mathcal{V}_{i j}$ of antennae $i$ and $j$ and model visibilities $\hat{\mathcal{V}}_{i j}$ according to $\epsilon^{2}=\sum\left|\mathcal{V}_{i j}-g_{i} g_{j}^{*} \hat{\mathcal{V}}_{i j}\right|^{2}$ for each of the correlations $L L$ and $R R$ (Schwab 1980; Sault et al. 2008). The model visibilities used are those found earlier. Note the subscripts here denote a specific antenna and not the polarization state as before.

4. The gain found from the continuum $L L$ data is then applied to the line $L L$ data, and similarly for the $R R$ continuum and line data.

5. The different visibilities $(L L, R R, R L, L R)$ are recombined and inverted to produce corrected and CLEANed maps. Spectra can be obtained either from the corrected visibilities or the maps.

Note that in the deconvolution steps (steps 2 and 5) the same instrumental beam is used for all polarizations. This is due to the behaviour of Miriad's invert, which produces a single instrumental beam corresponding to all image planes and Stokes parameters (sec. 13.4 of Sault et al. 2008). We checked that the instrumental response to different polarizations was similar by inverting the $L L$ - and $R R$-handed visibilities separately from one other and finding that the instrumental beams for both polarizations to be almost identical.

Figure 1 shows maps before- and after- correction maps for the continuum in Orion KL around $345 \mathrm{GHz}$. We see that before correction there are large peaks of Stokes $V$, and that the three (identified) positive peaks have a negative peak close by. After the correction, though there are still noisy Stokes $V$ signals throughout the image, the pairs of peaks have disappeared.

\section{OBSERVATIONS}

We collected radio interferometric polarimetry observations from the Submillimeter Array (SMA) archive that had been measured using the linear-to-circular QWP equipment, a similar setup to that used by Muñoz et al. (2012) to measure circular polarization from dust continuum in Sgr A*. Because the archival observations were not taken with measurements of $\mathrm{CP}$ in mind the SNR is often low, and we had to average velocity channels to increase it at the cost of spectral resolution. This generally increases the SNR from 3-4 to 6-10.

The four objects we present here are Orion KL, NGC7538, NGC1333 IRAS2A and IRC+10216. The first three are well-known star-forming regions, while IRC +10216 is an evolved carbon star. The data for Orion KL were previously used for a dust polarization study in Tang et al. (2010), and the data for IRC+10216 were previously used for spectral line polarimetry in Girart et al. (2012). The archival data for NGC1333
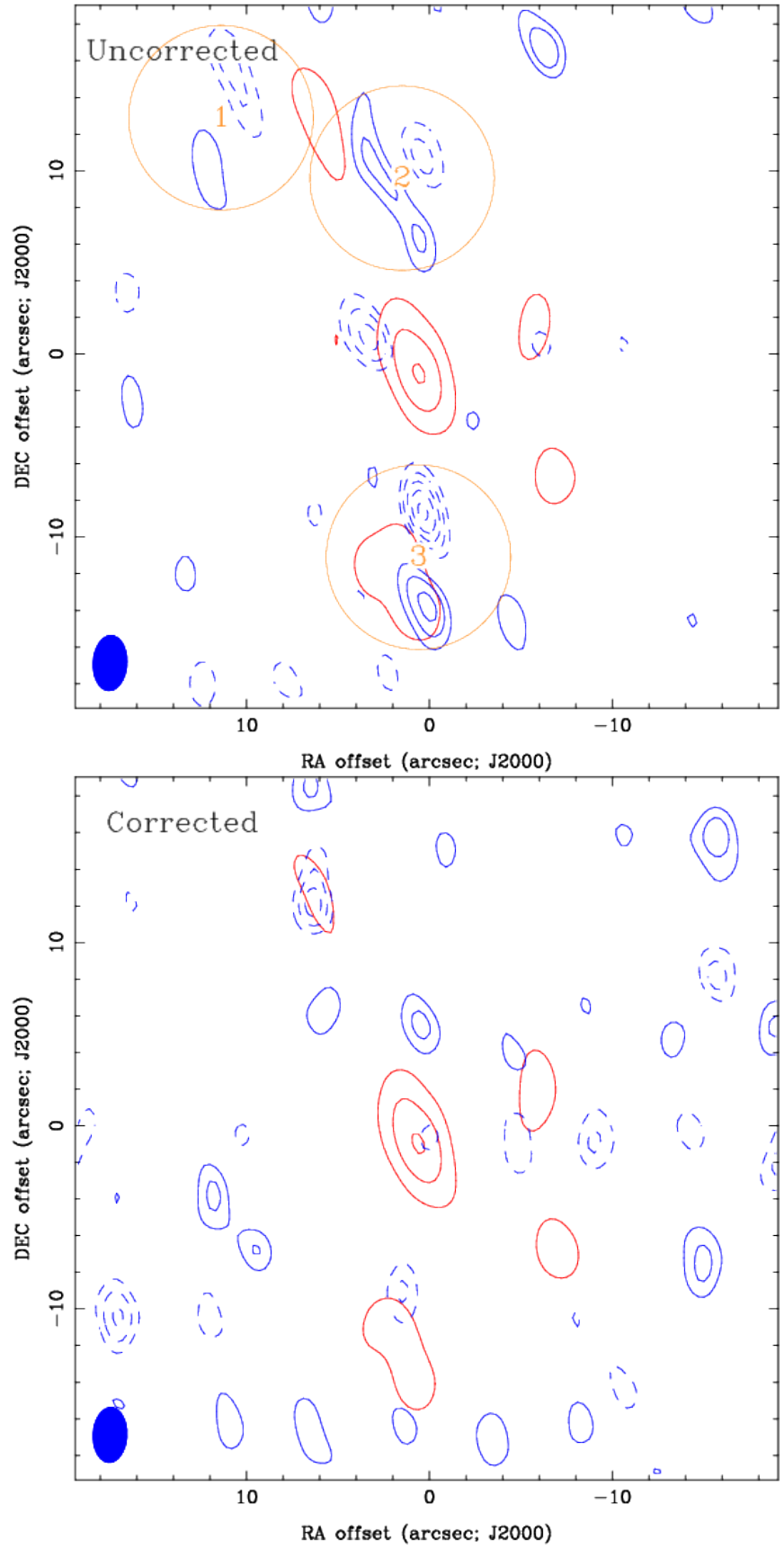

Figure 1. Map of the continuum around $345 \mathrm{GHz}$ in Orion KL before (top) and after (bottom) squint correction. Red contours are Stokes $I$, blue contours are Stokes $V$. Dashed lines denote negative values, solid lines denote positive values. Note the three pairs of positive and negative Stokes $V$ peaks in the uncorrected map (identified and circled in orange). These largely disappear after correction. Red Stokes $I$ contours are at $15 \%, 50 \%$, and $95 \%$ of the peak intensity. Blue Stokes $V$ contours are at -8, -7, -6, -5, -4, -3, -2, 2, 3, 4, $5,6,7$ and $8 \sigma$ levels.

IRAS2A and NGC7538 used here have not been published before as far as we are aware. We find significant Stokes $V$ signals in all objects except for NGC1333 IRAS2A. Table 1 shows a summary of the objects presented and related information. 


\begin{tabular}{llll}
\hline Object & $\begin{array}{l}\text { Coordinates } \\
(\mathbf{J} 2000)\end{array}$ & Array & Obs. Date \\
\hline \hline Orion KL & $\begin{array}{l}\text { RA } 05^{\mathrm{h}} 35^{\mathrm{m}} 14.501^{\mathrm{s}} \\
\text { Dec }-05^{\circ} 22^{\prime} 30.40^{\prime \prime}\end{array}$ & Compact & 2008-01-06 \\
\hline NGC7538 & $\begin{array}{l}\text { RA } 23^{\mathrm{h}} 13^{\mathrm{m}} 44.771^{\mathrm{s}} \\
\text { Dec }+61^{\circ} 26^{\prime} 48.85^{\prime \prime}\end{array}$ & Compact & $2014-10-28$ \\
\hline IRC+10216 RA: $09^{\mathrm{h}} 47^{\mathrm{m}} 57.381^{\mathrm{s}}$ & Compact & $2009-11-24$ \\
Dec $+13^{\circ} 16^{\prime} 43.70^{\prime \prime}$ & & \\
\hline NGC1333 & RA $03^{\mathrm{h}} 28^{\mathrm{m}} 55.580^{\mathrm{s}}$ & Compact & $2010-10-14$ \\
& Dec $+31^{\circ} 14^{\prime} 37.10^{\prime \prime}$ & & \\
\hline
\end{tabular}

Table 1. Summary of Archival Observations Used

The visibility data were corrected for beam squint, as explained in Section 3 in order to reduce spurious Stokes $V$ signals. As previously mentioned squint typically causes distinct pairs of positive and negative peaks of Stokes $V$ throughout the inverted image. The squint correction is confirmed visually by inspecting the Stokes $V$ maps, where we see the pairs of peaks largely disappear.

Figures 2 and 3 show corrected Stokes $I$ and Stokes $V$ spectra (left) obtained at the peak of the $\mathrm{CO}(J=3 \rightarrow$ 2) Stokes $V$ signal on the corresponding maps (right). A comparison of Stokes $V$ spectra before and after correction are shown in Figure 4. Notice in all cases the Stokes $V$ signal decreases after squint correction. Stokes $V$ can also be found in the average spectrum obtained from all the visibility data, though the SNR is of 3-5 $\sigma$ significance in that case compared to approximately $6-10 \sigma$ when the spectrum is taken at the peak of the inverted maps. The presence of a Stokes $V$ signal in the visibilities indicates the detections are not simply the result of the inversion process that creates the maps. That is, the Stokes $V$ signals are not due to sidelobes that appear when calculating the inverse Fourier transform of the visibilities. As can be seen in the maps, in general the peaks of Stokes $I$ and Stokes $V$ do not coincide.

In Orion KL (Fig. 2) the lines of $\mathrm{CO}(J=3 \rightarrow 2$ at $345.8 \mathrm{GHz})$ and $\mathrm{SiO}(J=8 \rightarrow 7$ at $347.3 \mathrm{GHz})$ are both bright (average visibilities show a peak Stokes $I$ of around $20 \mathrm{Jy} /$ beam and $55 \mathrm{Jy} /$ beam, respectively) and both show Stokes $V$ signals. The CO Stokes $V$ signal has an antisymmetric structure. On the other hand, Figure 5 shows that the peak $\mathrm{SiO}$ Stokes $V$ signal is purely negative. These lines (and others) are listed in Table 2 with their frequencies.

In IRC+10216 (Fig. 2) we again see Stokes $V$ in the $\mathrm{CO}(J=3 \rightarrow 2)$ but also in $\mathrm{CS}(J=7 \rightarrow 6)$, SiS $(J=19 \rightarrow 18)$, and $\mathrm{H}^{13} \mathrm{CN}(J=4 \rightarrow 3)$.

In NGC7538 (Fig. 3) the Stokes $V$ signal in CO $(J=3 \rightarrow 2)$ at $345.8 \mathrm{GHz}$ decreased in intensity after

\begin{tabular}{llcr}
\hline Object & Line & $(\mathbf{G H z})$ & $\begin{array}{c}\text { Stokes } V \\
(\mathbf{J y} / \text { beam })\end{array}$ \\
\hline \hline Orion KL & $\mathrm{CO}(J=3 \rightarrow 2)$ & 345.8 & 0.65 \\
\hline & $\mathrm{SiO}(J=8 \rightarrow 7)$ & 347.3 & -0.65 \\
\hline NGC7538 & $\mathrm{CO}(J=3 \rightarrow 2)$ & 345.8 & 0.85 \\
\hline IRC+10216 $\mathrm{CS}(J=7 \rightarrow 6)$ & 342.88 & 0.6 \\
\hline & $\mathrm{SiS}(J=19 \rightarrow 18)$ & 344.78 & 0.2 \\
\hline & $\mathrm{H}^{13} \mathrm{CN}(J=4 \rightarrow 3)$ & 345.34 & 0.8 \\
\hline & $\mathrm{CO}(J=3 \rightarrow 2)$ & 345.8 & 0.4 \\
\hline $\begin{array}{l}\text { NGC1333 } \\
\text { (IRAS2a) }\end{array}$ & $\mathrm{CO}(J=3 \rightarrow 2)$ & 345.8 & - \\
\hline
\end{tabular}

Table 2. Summary of corrected Stokes $V$ signals found. The beam size is determined by the configuration of the antennae array. An intensity for the peak of the Stokes $V$ signal is only given if the peak is noticeably higher than the noise level. The intensity quoted for CO in NGC7538 is before smoothing is applied.

correction but is still prominent. There was also initially a very strong Stokes $V$ signal in $\mathrm{CH}_{2} \mathrm{CO}$ at $346.6 \mathrm{GHz}$ that completely disappeared after correction.

Finally Figure 3 shows no detection in NGC1333, with only a clear detection of CO in Stokes $I$.

As mentioned previously, when assessing the Stokes $V$ detections we consult the map for obvious pairs of positive/negative peaks that would indicate beam offset and therefore a potentially false Stokes $V$ signal. The maps are integrated over a narrow frequency band of approximately $2 \mathrm{MHz}$ so any peaks that exist are not washed out by noise in adjacent channels. In the maps for Orion KL and IRC+10216 shown in Figure 2 there are no negative peaks around the peak of Stokes $V$. However in NGC7538 there is quite a large negative Stokes $V$ peak near our chosen peak that may indicate squint. The top panel of Figure 1 shows a typical appearance for squint signals which disappear after correction, and the pairs tend to resemble each other in shape. The pair of peaks around our chosen peak in NGC7538 however have distinct shapes. The worst case here is that the signal is entirely squint but on the other hand the signal may be a mixture of real and heavily affected by squint. The detections in Orion KL and IRC+10216 are more reliable.

\section{DISCUSSION}

The first question to address is whether our CP detections are real or result from instrumental artifacts. This is our chief concern because of the difficulty in calibrating $\mathrm{CP}$ measurements, especially since the observations presented here were not made with any special consid- 

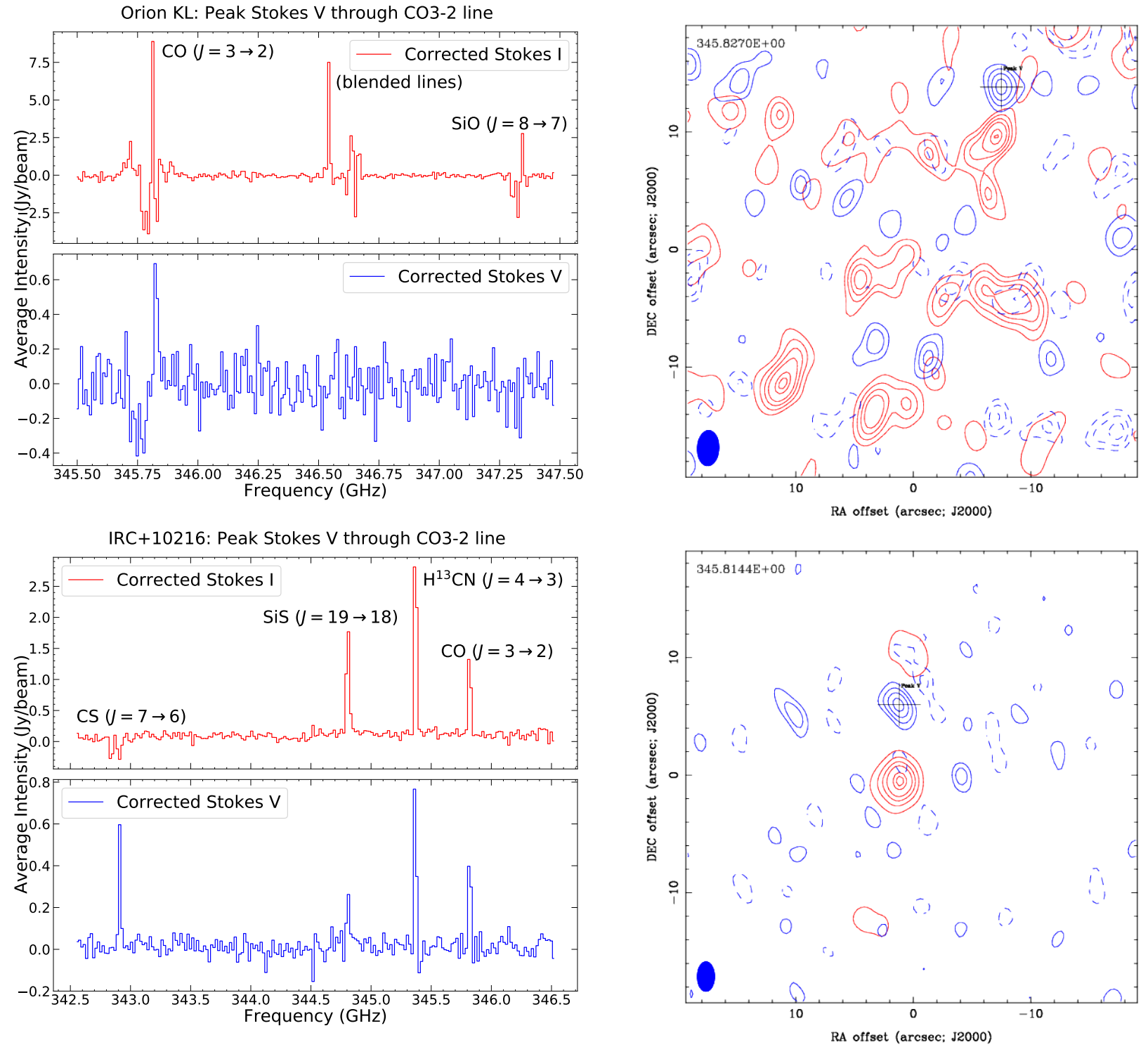

Figure 2. Corrected spectra and maps of the CO $(J=3 \rightarrow 2)$ line $(345.8 \mathrm{GHz})$ for Orion KL (top) and IRC+10216 (bottom). Spectra: Miriad's maxfit is used on the CO map to obtain the location on the image where the Stokes $V$ signal at $345.8 \mathrm{GHz}$ is maximum, and a spectrum is obtained through that point. The cross on the map denotes the location of the Stokes $V$ peak. The red line is Stokes $I$ and the blue is Stokes $V$. Maps: Blue contours are Stokes $V$ and are shown at the $-4,-3,-2,2,3,4 \sigma$ levels. The RMS error for each Stokes $V$ map is found using Miriad's imstat command: $\sigma=0.30$ and 0.17 Jy/beam, for Orion KL and IRC+10216, respectively. The distance of the peak to the phase center is 15 " and 6", respectively. Dark red contours are Stokes $I$ and the levels are 15\%,30\%,45\%,60\%, $85 \%$ and $95 \%$ of the maximum. The value in the top left is the central frequency in $\mathrm{GHz}$ of the mapped signal, integrated over a narrow bandwidth of $\sim 2 \mathrm{MHz}$.

erations for calibrating $\mathrm{CP}$ as in the observation of Sgr A* reported in Muñoz et al. (2012). We will repeat here the arguments made in Section 4 in support for the soundness of these detections, and then discuss earlier detections of $\mathrm{CP}$ and summarize how ARS can explain the detections presented in this work.

Firstly, we take the average of all the visibility data (not shown) and note that the peak Stokes $V$ is not proportional to the peak Stokes $I$ at any particular frequency. For example, a large Stokes $I$ at $347.25 \mathrm{GHz}$ $(\mathrm{SiO}(J=8 \rightarrow 7))$ does not indicate a corresponding peak in Stokes $V$. This property indicates that there is no systematic leakage of Stokes $I$ into Stokes $V$, however excluding the longest baselines from the averaging does cause the Stokes $V$ signals to follow Stokes $I$, pointing to some instrumental $\mathrm{CP}$ in the shortest baselines.

In the average visibilities (not shown) of Orion KL $\mathrm{CO}(J=3 \rightarrow 2)$ and $\mathrm{SiO}(J=8 \rightarrow 7)$ are the strongest lines, with the $\mathrm{SiO}(J=8 \rightarrow 7)$ line the stronger. Therefore, if the Stokes $V$ signal were purely leakage from Stokes $I$ then we would expect to see an SiO Stokes $V$ signal that is stronger than the CO Stokes $V$ signal in the visibilities, but we do not; the Stokes $V$ signal from $\mathrm{CO}(J=3 \rightarrow 2)$ is stronger. This is also true in the visibilities of IRC+10216, where the CS $(J=7 \rightarrow 6)$ and $\operatorname{SiS}(J=19 \rightarrow 18)$ lines have similar strengths but the Stokes $V$ at $\operatorname{SiS}(J=19 \rightarrow 18)$ is twice as intense. However, in the same object $\mathrm{H}^{13} \mathrm{CN}(J=4 \rightarrow 3)$ and 

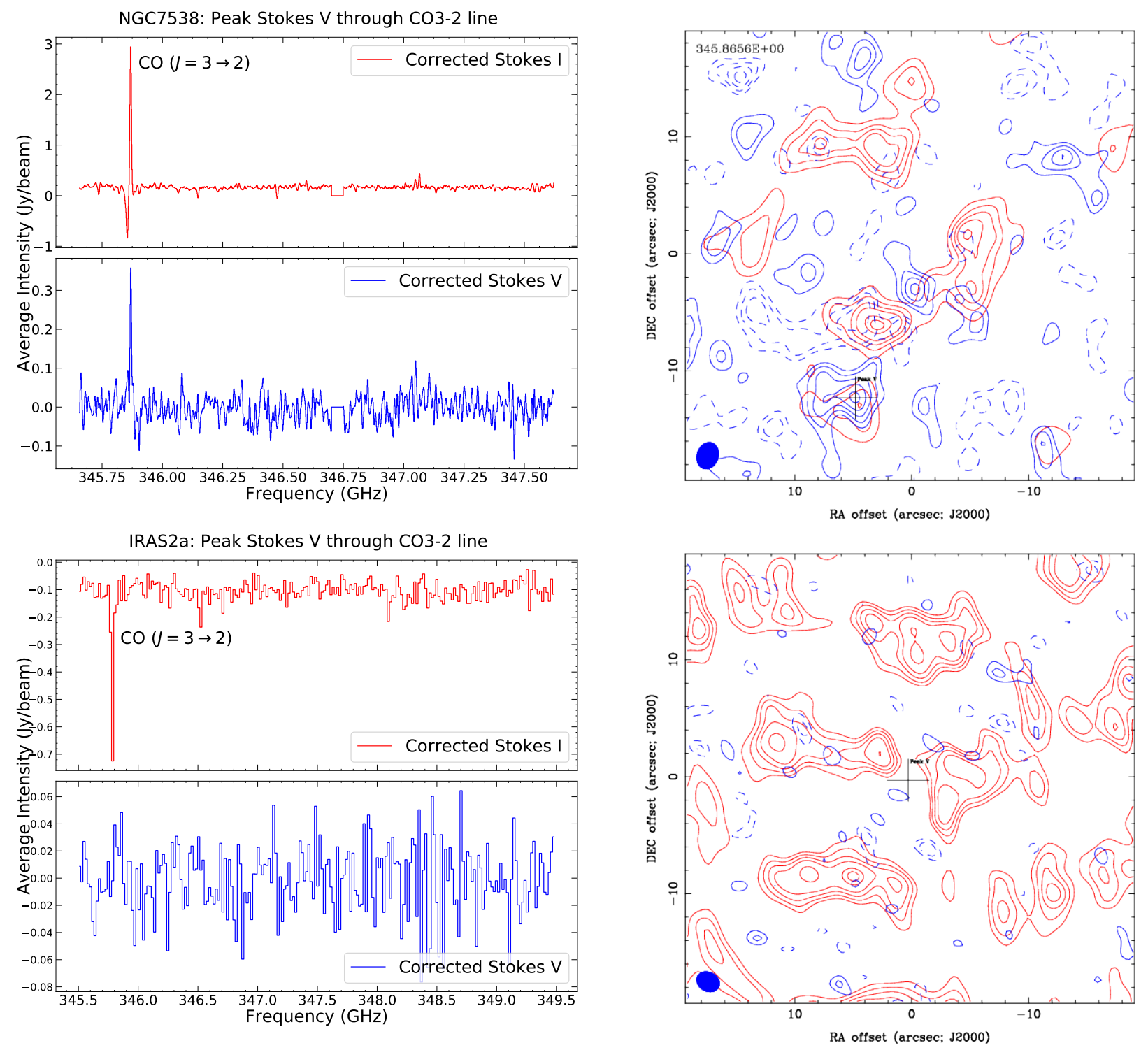

Figure 3. Same as Figure 2 but for NGC7538 (top) and NGC1333 IRAS2A (bottom). The spectrum for NGC7538 is Hanning smoothed. No significant Stokes $V$ signal is detected in NGC1333 IRAS2A, probably because the object is too dim. Contours are the same levels as in Figure 2 and the spectrum is obtained the same way. The RMS for the NGC7538 and NGC1333 IRAS2A maps is $\sigma=0.15$ and $0.12 \mathrm{Jy} /$ beam, respectively. The distance of the peak to the phase center is 13 " for NGC7538.

$\mathrm{CO}(J=3 \rightarrow 2)$ have Stokes $V$ intensities that appear proportional to their Stokes $I$ intensity (i.e., stronger $I$ means stronger $V$ ).

We also note that for Orion KL the shapes of the Stokes $V$ signals vary across the frequency band and interpret this to mean that the signals are likely not instrumental in nature, assuming that any instrumental mechanism for producing spurious Stokes $V$ produces a single type of CP (left or right). For example Figure 5 shows a spectrum for that source with Stokes $V$ in $\mathrm{CO}$ and $\mathrm{SiO}$. The $\mathrm{SiO}$ signal is purely negative (indicating only LCP) but the CO signal is antisymmetric, indicating the presence of both LCP and RCP. This has a physical explanation using the ARS model in terms of blue-shifted and red-shifted scattering populations that will be considered in Section 5.2. We know of no instrumental mechanism for producing such a signature. For the other objects the Stokes $V$ signal is always positive.

In the case of IRC+10216, an evolved carbon star with an extended envelope, the peak of Stokes $V$ in the CO $(J=3 \rightarrow 2)$ map (bottom-right panel of Figure 2) is approximately 6 " away from the Stokes $I$ emission, which at first glance seems to point to an erroneous detection. However the real size of the CO envelope around IRC +10216 is much larger than seen here, as can be verified from single-dish $\mathrm{CO}(J=2 \rightarrow 1)$ observations showing a circumstellar shell with a radius of about 50" (Fig. 1 of Cernicharo et al. 2015).

Spatial filtering due to the resolution of the interferometer explains the smaller spatial extent of IRC +10216 in the observations presented here and also explains the frequent occurences of negative Stokes $I$ in almost all the spectra shown in Figures 2, 3, and 5, as well as the high levels of CP ranging from $6 \%$ to $30 \%$. The largest 

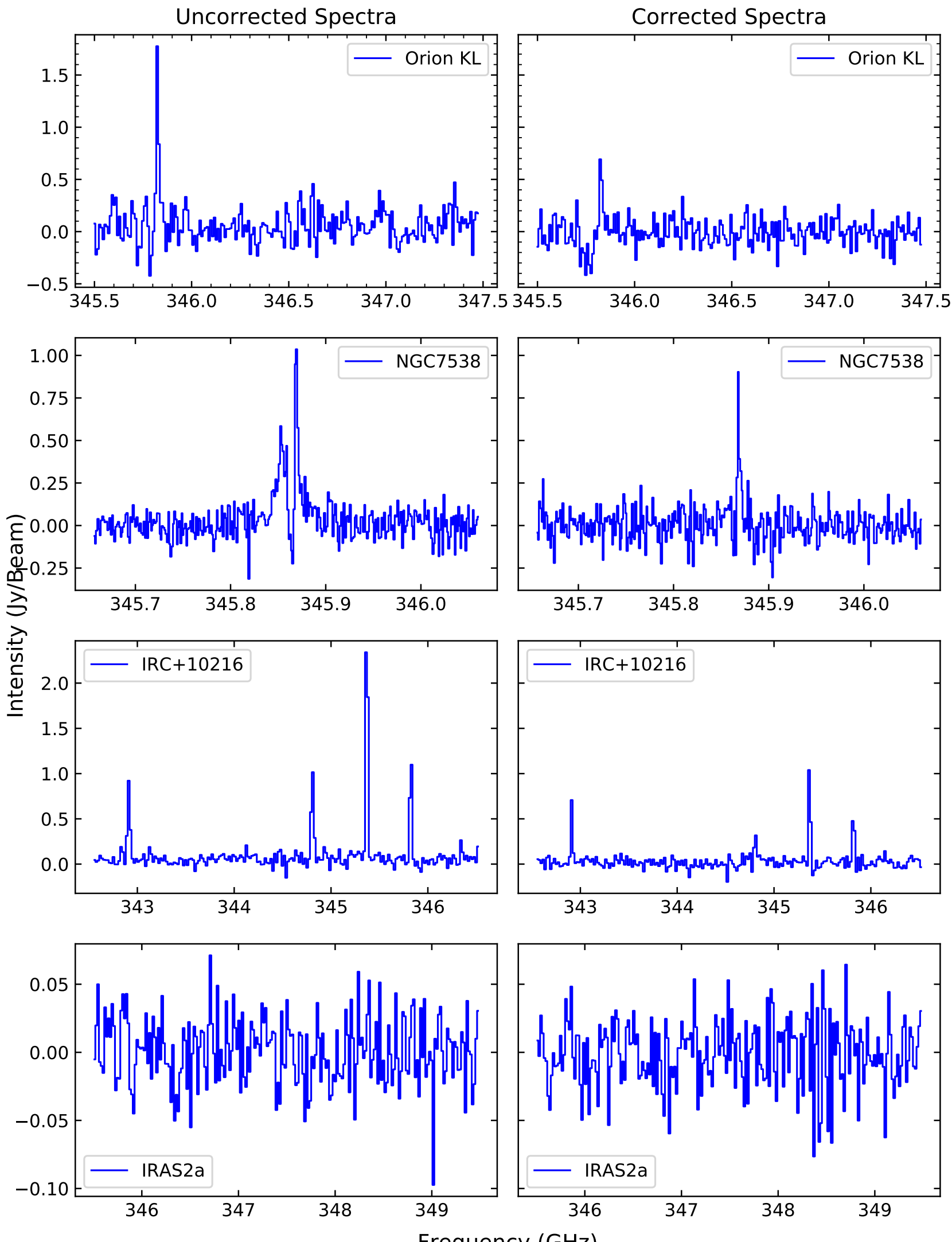

Frequency $(\mathrm{GHz})$

Figure 4. Stokes $V$ spectra of all objects before and after squint correction. Miriad's maxfit is used on the CO map for each object to obtain the location in the image where the Stokes $V$ signal at $345.8 \mathrm{GHz}$ is maximum, and a spectrum is obtained through that point. Note that the Stokes $V$ signal decreases in all cases after squint correction. The location of the peak varies: sometimes it moves closer to the phase center after correction, sometimes further away. 


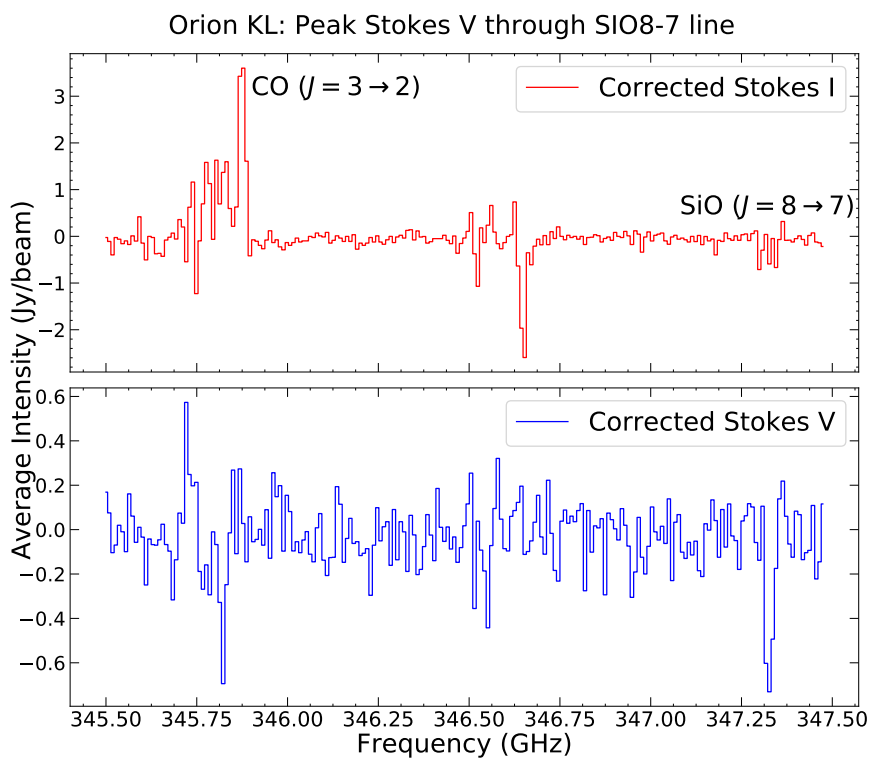

Figure 5. Peak Stokes $V$ signal for the $\mathrm{SiO}(J=7 \rightarrow 8$ at $347.3 \mathrm{GHz}$ ) transition in Orion KL. Note there is also a strong Stokes $V$ signal in the CO $(J=3 \rightarrow 2$ at $345.8 \mathrm{GHz})$ transition here. The $\mathrm{SiO}$ signal is purely negative but the $\mathrm{CO}$ signal is antisymmetric.

resolvable scale by an interferomter is determined by the length of the shortest baseline, meaning that large scale emission can be filtered out by the interferometer. For example in Orion $\mathrm{KL}$ the $\mathrm{CO}(J=3 \rightarrow 2)$ Stokes $I$ emission is large and extended (Hull et al. 2014). If the Stokes $V$ signal came from smaller localized areas, then we would observe peaks of Stokes $V$ as shown while only a portion of the Stokes $I$ would be present. The rest of the Stokes $I$ signal would be filtered away, which could shift the zero-level downward. Fluctuations in Stokes $I$ could then appear to have negative values, while the weaker Stokes $I$ could also explain the high levels of $V / I$ observed.

Since the Stokes $V$ features shown in the maps of Orion KL and IRC+10216 are compact we made maps excluding the shortest baselines, which have instrumental CP as shown from the averaging of the visibilities, and included only the longest baselines. If they are real, we expect the compact features to remain in these maps since the longest baselines have the highest resolution. We saw that the Stokes $V$ peak remains in the maps of IRC+10216 after excluding more than half of the baselines as expected, however, the signal in Orion KL appears to disappear after excluding the shortest half of the baseline distances. This may indicate that the peak shown in Orion KL in Figure 2 is instrumental.

The level of instrumental CP is in general greater the further the peak is from the center of the map since the instrumental polarization is found at the phase center. The peaks shown for Orion KL and NGC7538 (Figures
2 and 3) are several FWHMs of the beam away from the center, meaning the level of instrumental contamination in those peaks is likely higher.

Finally, we checked and confirmed that there were no Stokes $V$ signals in the continuum comparable to those found in molecular lines like $\mathrm{CO}$ and $\mathrm{SiO}$. In the continuum, $V / I$ was found to be $<1 \%$ for Orion $\mathrm{KL}$ and IRC +10216 , and $<4 \%$ in NGC7538. If the detected Stokes $V$ originated from instrumental artifacts (other than $I$ leakage) then we would expect to detect Stokes $V$ at similar levels in the continuum, but this is not seen. The level of Stokes $V$ found in molecular transitions is always higher than the level of Stokes $V$ in the continuum for the observations presented here, and no significant Stokes $V$ is found in the continuum.

We feel confident that the CP reported here, although perhaps suffering from some level of instrumental contamination, is real and originates from within these objects.

\subsection{Earlier Non-Zeeman CP Detections}

$\mathrm{CP}$ in a molecular spectral line weakly sensitive to the Zeeman effect was first reported by Houde et al. (2013), where approximately $2 \%$ polarization was detected in the ${ }^{12} \mathrm{CO}(J=2 \rightarrow 1)$ transition at $230.5 \mathrm{GHz}$ in Orion KL using the FSPPol at the CSO. The CP signal was approximately symmetric (i.e. " $\cap "$-shaped). The observation was repeated three months after the first measurement to confirm the result was not spurious, with similar results. Additionally the strong line of $\mathrm{HCN}(J=3 \rightarrow 2)$ at $265.9 \mathrm{GHz}$ in Orion $\mathrm{KL}$ was measured and no CP higher than the $0.1 \%$ level was detected. The detection in $\mathrm{CO}$ and the absence of a detection in $\mathrm{HCN}$ were evidence that the FSPPol/CSO observations were not significantly suffering from leakage into Stokes $V$ and highlighted the CO molecule as a target for non-Zeeman CP. In all the objects presented here we find $\mathrm{CP}$ in ${ }^{12} \mathrm{CO}(J=3 \rightarrow 2)$ at $345.8 \mathrm{GHz}$ (except for in NGC1333 IRAS2A where the CO line is relatively weak). This is consistent with the original 2013 detection.

In follow up work Hezareh et al. (2013) examined the supernova remnant IC 443 using dust polarimetry with PolKa at APEX and polarization maps of ${ }^{12} \mathrm{CO}(J=$ $2 \rightarrow 1)$ and $(J=1 \rightarrow 0)$ taken with the IRAM $30 \mathrm{~m}$ telescope. They initially found that the LP maps of dust and CO differed greatly in their polarization angles. Expecting that there was conversion of linear to circular polarization due to ARS, the CO Stokes $V$ fluxes were then reinserted into the CO LP signals. The resulting $\mathrm{CO}$ polarization angle maps then agreed very well with each other, as well as with the dust map (Fig. 9 of Hezareh et al. 2013). This result clearly establishes a conversion from linear to circular polarization. 
The detections of $\mathrm{CP}$ in $\mathrm{SiO}(\nu=1$ and $\nu=2$, $J=1 \rightarrow 0$ ) masers at 43.1 and $42.8 \mathrm{GHz}$, respectively, were observed to have line profiles inconsistent with the Zeeman effect (Cotton et al. 2011). An attempt to explain the Stokes $V$ shapes with a non-Zeeman mechanism involving the anisotropic pumping of the masers and a varying magnetic field along the line of sight put forward by Wiebe \& Watson (1998) could not account for the high levels of CP observed (Cotton et al. 2011). We note that these $\mathrm{SiO}$ maser observations underwent a similar self-calibration based process to correct for beam squint as the observations presented here.

\subsection{Anisotropic Resonant Scattering}

ARS was the mechanism first proposed by Houde et al. (2013) to explain the presence of CP in the transitions of CO. ARS rests on a second-order interaction between radiation and matter in the presence of a magnetic field. Incident radiation with photon states polarized $\|$ and $\perp$ to the magnetic field can scatter slightly differently off a molecule and incur a small phase shift between the photon states. The phase shift incurred after propagating and scattering off many molecules results in the appearance of $\mathrm{CP}$ in the scattered radiation.

While this mechanism could reproduce the level of $\mathrm{CP}$ observed in $\mathrm{CO}$ it initially failed to explain the observed symmetric " $\cap$ "-shaped Stokes $V$ profile. In a follow up paper Houde (2014) considered the observations of Stokes $V$ in $\mathrm{SiO}$ masers (Cotton et al. 2011) and showed that the different profile shapes detected are readily explained through ARS off populations of foreground molecules located slightly outside of the velocity range of the line. For example, a blue-shifted scattering population of molecules could result in a negative " $U$ "shaped profile while a red-shifted population in a positive " $\cap$ "-shaped profile. The presence of both a blueand red-shifted population would cause an antisymmetric "S"-shaped profile (like the one seen in the top left panel of Figure 2).

The conversion of LP to CP due to ARS can be illustrated by considering background LP radiation oriented at some angle $\theta$ to the foreground magnetic field. The incident and scattered radiation can be written in terms of the $n$-photon states as (Houde et al. 2013)

$$
\begin{aligned}
|\psi\rangle & =\alpha\left|n_{||}\right\rangle+\beta\left|n_{\perp}\right\rangle \\
\left|\psi^{\prime}\right\rangle & \simeq \alpha e^{i \phi}\left|n_{||}\right\rangle+\beta\left|n_{\perp}\right\rangle,
\end{aligned}
$$

where $\alpha=\cos (\theta), \beta=\sin (\theta)$ and $\phi$ is a phase shift incurred after multiple scattering events. Following the definitions of the Stokes parameters and using an appropriate basis the Stokes parameters for the scattered radiation can be found to be

$$
I=\alpha^{2}+\beta^{2}
$$

$$
\begin{aligned}
& Q=\alpha^{2}-\beta^{2} \\
& U=2 \alpha \beta \cos (\phi) \\
& V=2 \alpha \beta \sin (\phi) .
\end{aligned}
$$

This implies that in the chosen basis Stokes $U$ is lost to Stokes $V$, i.e., more generally, LP is converted to CP. A calculation of the phase shift $\phi$ incurred due to ARS can be found in Houde et al. (2013).

Now, given a conversion from $U$ to $V$ it is clear that measuring $V$ is necessary for techniques like the DCF method that rely on the dispersion of the PAs of LP to calculate the strength of the magnetic field. This is because without corresponding $V$ measurements, the PAs obtained from a molecular spectral line subject to ARS (like CO $(J=3 \rightarrow 2)$ ) will be rotated according to

$$
\tan (2 \chi)=\cos (\phi) \tan \left(2 \chi_{0}\right),
$$

where $\chi_{0}$ is the PA of the incident radiation and $\phi$ is the incurred phase shift as before (eq. 10 of Houde 2014), changing the dispersion of the PAs, as seen in Hezareh et al. (2013). As mentioned earlier, LP to CP conversion was reversed to obtain corrected polarization angles.

Because the DCF method relies on the observed dispersion of PAs using LP, any such studies using molecular lines must also include corresponding CP measurements to account for the polarization conversion effect and determine the correct orientation of the PAs (Hezareh et al. 2013; Chandrasekhar \& Fermi 1953; Hildebrand et al. 2009; Houde et al. 2009).

\section{CONCLUSION}

We analyzed polarimetric observations from the SMA archive of Orion KL, IRC+10216, NGC7538 and NGC1333 IRAS2A to search for CP signals. The data were corrected for squint, a source of spurious Stokes $V$ signals that arises due to a slight misalignment in the beams used to obtain Stokes $V$ when performing observations. We found evidence of significant Stokes $V$ in Orion $\mathrm{KL}($ in $\mathrm{CO}(J=3 \rightarrow 2)$ and $\mathrm{SiO}(J=8 \rightarrow 7))$, in $\mathrm{IRC}+10216$ (in $\mathrm{CS}(J=7 \rightarrow 6)$, $\operatorname{SiS}(J=19 \rightarrow 18)$, $\mathrm{H}^{13} \mathrm{CN}(J=4 \rightarrow 3)$ and $\left.\mathrm{CO}(J=3 \rightarrow 2)\right)$ and in NGC7538 (in CO $(J=3 \rightarrow 2)$ ). We measured relatively important levels of CP ranging from 6\%-30\%, probably due to the interferometric spatial filtering of large scale emission. No significant Stokes $V$ was found in the continuum of any of the objects.

Theories that explain the presence of non-Zeeman CP in molecular spectral lines rely on the conversion of background LP to CP. The detections in multiple lines and objects presented here indicate that such an effect is likely widespread. Since the conversion of LP-to-CP modifies the observed dispersion of PAs, it is necessary to obtain precise measurements of CP along with LP for corresponding studies of the magnetic field in the 
interstellar medium.

The Submillimeter Array is a joint project between the Smithsonian Astrophysical Observatory and the Academia Sinica Institute of Astronomy and Astrophysics and is funded by the Smithsonian Institution and the Academia Sinica. M.H. is supported by the Natural Science and Engineering Research Council of
Canada Discovery Grant RGPIN-2016-04460. J.M.G is supported by the MINECO (Spain) AYA2014-57369-C3 and AYA2017-84390-C2 grants.

Reduction scripts are available at http://github. com/mef51/SMAData. A Python wrapper for Miriad including the squint correction script used is available at http://github.com/mef51/smautils.

\section{REFERENCES}

Cernicharo, J., Marcelino, N., Agúndez M., Guéllin, M. 2015 A\&A, 575, A91

Chandrasekhar, S., Fermi, E. 1953 ApJ, 118, 113

Cortes, P. C., Crutcher, R. M., Watson, W. D. 2005, ApJ, 628, 780 .

Cotton, W. D., Ragland, S., Danchi, W. C. 2011, ApJ, 736, 96

Crutcher, R. M. 2012 Annu. Rev. Astron. Astrophys, 50, 29-63

Davis, L., Jr. 1951, PhRv, 81, 890

Girart, J. M., Crutcher, R. M., Rao, R. 1999, ApJ, 525, L109.

Girart, J. M., Patel, N., Vlemmings, W. H. T., Rao, R. 2012, ApJ, 751, L20.

Glenn, J., Walker, C. K., Bieging, J. H., et al. 1997, ApJ, 487, L89.

Goldreich, P., Kylafis, N. D. 1981 ApJ, 243, L75-L78

Greaves, J. S., Holland, W. S., Friberg, P., et al. 1999, ApJ, 512, L139.

Hamaker, J. P., Bregman, J. D., Sault, R. J. 1996 A\&A Suppl. Ser., 117, 137-147

Hezareh, T., \& Houde, M. 2010, PASP, 122, 786

Hezareh, T. J., Wiesemeyer, H., Houde, M., Gusdorf, A., Siringo, G. 2013 A\&A, 558, A45

Hildebrand, R. G., Kirby, L., Dotson. J. L., Houde, M., Vaillancourt, J. E. 2009 ApJ, 696, 567-573

Houde, M., Vaillancourt, J. E., Hildebrand, R. H., Chitsazzadeh, S., Kirby, L., 2009 ApJ, 706, 1504

Houde, M., Hezareh, T., Jones, S., Rajabi, F. 2013 ApJ, 764, 24
Houde, M. 2014 ApJ, 795, 27

Hull, C. L. H., Plambeck, R. L., Kwon, W., Bower, G. C., Carpenter, J. M., Crutcher, R. M., et al. 2014, ApJSuppl., 213, 13

Hunter, J. D. 2007, Matplotlib: A 2D Graphics Environment, Computing in Science \& Engineering, 9, 3, 90-95

Muñoz, D. J., Marrone, D. P., Moran, J. M., Rao, R. 2012 ApJ, 745,115

Marrone D. P., Rao R. 2008, Proc. SPIE 7020, 70202B

Lai, S.-P., Girart, J. M., Crutcher, R. M. 2003, ApJ, 598, 392.

Sault, R. J., Teuben, P. J. Wright, M. C. H. 1995 ADASS IV, ASP Conference Series, 77, 433-436

Sault, R. J., Hamaker, J. P., Bregman, J. D. 1996 A\&A Suppl. Ser., 117, 149-159

Sault, R. J., Killeen, N. 2008, Miriad Users Guide, Australia Telescope National Facility

Schwab, F. R. 1980, Proc. SPIE 0231, Intl Optical Computing Conf I

Tang, Y.-W., Ho, P. T. P., Koch, P. M., Rao, R. 2010, ApJ, 717, 1262

Thompson, A. R., Moran, J. M., Swenson Jr, G. W. 2001, Interferometry and synthesis in radio astronomy (John Wiley \& Sons)

Wiebe, D. S., Watson, W. D. 1998, ApJ, 503:L71-L74 